\title{
3D Reconstruction from Projection Matrices in a C-Arm Based 3D-Angiography System
}

\author{
N. Navab ${ }^{1}$, A. Bani-Hashemi ${ }^{1}$, M. S. Nadar ${ }^{1}$ \\ K. Wiesent ${ }^{2}$, P. Durlak ${ }^{2}$, T. Brunner ${ }^{2}$, K. Barth ${ }^{2}$ and R. Graumann ${ }^{2}$ \\ 1 Siemens Corporate Research, Inc. \\ 755 College Road East \\ Princeton, NJ 08540 \\ navab@scr.siemens.com \\ 2 Siemens AG, Medical Engineering Group \\ Erlangen, Germany
}

\begin{abstract}
D reconstruction of arterial vessels from planar radiographs obtained at several angles around the object has gained increasing interest. The motivating application has been interventional angiography. In order to obtain a three-dimensional reconstruction from a $\mathrm{C}$-arm mounted X-Ray Image Intensifier (XRII) traditionally the trajectory of the source and the detector system is characterized and the pixel size is estimated. The main use of the imaging geometry characterization is to provide a correct 3D-2D mapping between the 3D voxels to be reconstructed and the $2 \mathrm{D}$ pixels on the radiographic images.

We propose using projection matrices directly in a voxel driven backprojection for the reconstruction as opposed to that of computing all the geometrical parameters, including the imaging parameters. We discuss the simplicity of the entire calibration-reconstruction process, and the fact that it makes the computation of the pixel size, source to detector distance, and other explicit imaging parameters unnecessary.

A usual step in the reconstruction is sinogram weighting, in which the projections containing corresponding data from opposing directions have to be weighted before they are filtered and backprojected into the object space. The rotation angle of the $\mathrm{C}$-arm is used in the sinogram weighting. This means that the C-arm motion parameters must be computed from projection matrices. The numerical instability associated with the decomposition of the projection matrices into intrinsic and extrinsic parameters is discussed in the context. The paper then describes our method of computing motion parameters without matrix decomposition. Examples of the calibration results and the associated volume reconstruction are also shown.
\end{abstract}

\section{Background and Justification}

Interventional angiography has motivated many research and development work on $3 \mathrm{D}$ reconstruction of arterial vessels from planar radiographs obtained at several angles around the subject. The endovascular therapy of subarachnoid aneurysms using detachable Guglielmi coils is an application where an imaging 
system with 3D-capabilities is very useful, [4]. The success of this intravascular procedure critically depends on the packing of the aneurysm. Coils projecting into parent vessels may cause thrombosis, while incomplete filling may lead to regrowth of the aneurysm. Other applications that would benefit from the $3 \mathrm{D}$ capabilities are the treatment of carotid stenoses and high resolution bone imaging.

In order to obtain a three-dimensional reconstruction from a $\mathrm{C}$-arm mounted XRII traditionally the trajectory of the source and detector system is characterized and the pixel size is computed. Variety of methods have been proposed in the literature to compute different sets of characterization parameters [15], [2], [4], [3]. Rouge et al. [15] use a non-linear minimization method to compute the position and orientation of the $\mathrm{X}$-ray system relative to the world coordinate system, as well as the image center and scale, using a spiral phantom. Koppe et al. [2] use two consecutive calibration steps to compute 15 parameters explicitly. These parameters are the source position, iso-center, focus parameters, position and orientation. The main use of the imaging geometry characterization is however to provide a correct 3D-2D mapping between the $3 \mathrm{D}$ voxels to be reconstructed and the $2 \mathrm{D}$ pixels on the radiographic images. We suggest a simple calibration procedure that results in the 3D-2D mapping, as well as, the backprojection algorithm that uses this mapping. As a result we avoid explicit computation of quantities such as pixel size, source to detector distance, and the scale factors due to image digitization. This makes the whole calibrationreconstruction process simple and accurate. An X-ray calibration phantom can be positioned around the head of the patient. This will only occupy a small portion of the X-ray image. The projection matrices are then easily computed. These matrices are then directly used for the back-projection. There is then no need for decomposing these matrices into different geometry and imaging parameters.

If we use the projection matrices computed using an X-ray calibration phantom for 3D reconstruction, the result will be in the coordinate system attached to the calibration phantom. It is however important to present the reconstructed volume in a coordinate frame that is intuitive and natural for the clinicians. During the interventional procedures the $\mathrm{C}$-arm is often moved in the cranial to caudal and LAO to RAO directions to find an optimum view of the lesion. One of the main advantages of this $3 \mathrm{D}$ reconstruction is the off-line examination of the vessel tree in order to define an optimum view. This will minimize the radiation exposure to the patient, as well as, the injection of contrast material. Another factor in interventional neuroradiology is the proper placement of the patient to target an organ or a lesion for imaging. An intuitive reference frame is needed for proper placement of patient prior to data collection.

The iso-center of the $\mathrm{C}$-arm is a natural choice, and the effective (average) rotation axis of the gantry is also a natural way to describe a principle axis in the equipment coordinate frame. Therefore, the $3 \mathrm{D}$ reconstruction must be presented in this coordinate frame. The matrices mapping $3 \mathrm{D}$ voxels to $2 \mathrm{D}$ projections must now map the voxels from the $\mathrm{C}$-arm coordinate frame to the projection plane. This makes it necessary to compute the motion of the C-arm. Due to 
numerical instabilities of matrix decomposition, it is desired to compute this motion directly without matrix decomposition. This is done quite accurately, and new mathematical formulation for the use of Quaternion representation is introduced [12].

We show that parameters such as X-ray source position and intra-frame motion of the imaging system can be computed without the decomposition of the projection matrix. A series of X-ray source positions result in a mean isocenter and a mean axis of rotation. This helps define an intuitive coordinate system at the iso-center of the $\mathrm{C}$-arm. The mean axis of rotation defines one of the principle axes.

The only assumption made is that the intrinsic parameters do not vary between two consecutive frames. Due to small angular motion of the C-arm and the normalization done through distortion correction process $[10,5]$, this assumption is in practice always satisfied.

We suppose that the image intensifier is free of distortion effect. This is in our application done through an off-line distortion characterization and an on-line distortion correction. The coming generation of X-ray angiography systems use solid state detectors. These detectors are free of geometric distortion, so in the future the distortion correction step will be unnecessary.

\section{Definition of Projection Geometry}

The 3D-2D mapping is represented by $\mathbf{P}$ a $3 \times 4$ homogeneous matrix of projection. This matrix can be computed by imaging a known phantom and establishing correspondences between feature points on the phantom and their radiographic image. The projection matrix $\mathbf{P}$ is defined up to a scale factor. This matrix represents all the imaging geometry parameters. These parameters can be divided into two sets. The first set is called the extrinsic parameters. These parameters define the position and orientation of the imaging system in a world coordinate system, e. g. the coordinate system associated with the calibration phantom. The second set of parameters is called the intrinsic parameters. These parameters only depend on internal parameters of our radiographic imaging system such as pixel size, image center, and source to detector distance.

The imaging system is modeled after a simple pinhole camera. This model proves to be sufficiently accurate for this application. A C-arm coordinate system is defined with its origin at the $\mathrm{X}$-ray source. We define the $\mathrm{z}$-axis parallel to the normal dropping from $\mathrm{X}$-ray source onto the image plane. The $\mathrm{x}$-axis and $\mathrm{y}$-axis are parallel to the row and column vectors of the $2 \mathrm{D}$ detector plane.

The homogeneous matrix $\mathbf{P}$ maps voxels from the $\mathrm{C}$-arm coordinate frame to the image plane. We have:

$$
\mathbf{P} \cong\left[\begin{array}{ll}
\mathbf{A R} & \mathbf{A T}
\end{array}\right]
$$

where $\mathbf{A}\left[\begin{array}{ccc}\alpha_{u} & 0 & u_{0} \\ 0 & \alpha_{v} & v_{0} \\ 0 & 0 & 1\end{array}\right]$ 


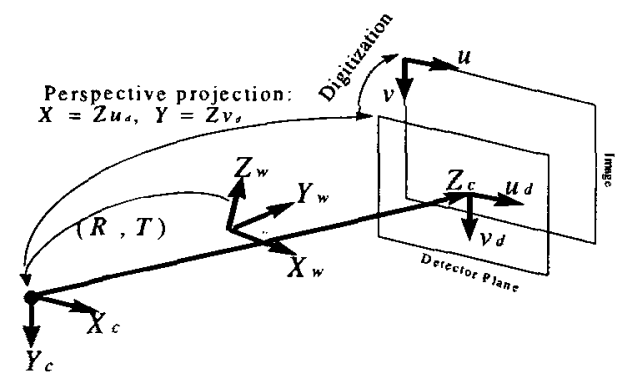

Fig. 1. Mapping between different coordinate systems

The symbol $\cong$ is used to emphasize that the equality is in homogeneous coordinates and therefore up to scale. The parameters defining $\mathbf{R}$, and $\mathbf{T}$ are called the extrinsic parameters. The matrix $\mathbf{A}$ incorporates scaling along the horizontal and vertical directions. It also incorporates shifting of the coordinate center from the image center (intersection of optical axis with the image plane) to any arbitrary point on the image. The parameters $\alpha_{u}$ and $\alpha_{v}$ also incorporate in them any horizontal and vertical scale changes due to the digitization process. They also include the changes due to the increase or decrease of the relative distance between source and detector. It is important to note that the matrix $\mathbf{P}$ relates every 3D point in the world coordinate frame to a pixel in the image. Figure 1 illustrates the imaging process including: (a) going from a point in the world coordinate system $\left(X_{w}, Y_{w}, Z_{w}\right)$ to a $\mathrm{C}$-arm coordinate system $\left(X_{c}, Y_{c}, Z_{c}\right)$, (b) perspective projection onto the detector plane, and (c) the digitization process and change of the coordinate center to an arbitrary point (upper left corner).

In the traditional computed tomography reconstruction, the motion of the $\mathrm{C}$-arm, the relation between the $\mathrm{X}$-ray source and the image intensifier, and the pixel size are computed in different steps. These quantities are later used in the mathematical formulation of the back-projection geometry, from image coordinates in pixels to 3D coordinates in voxels (for example, in millimeters). Here we directly compute the projection matrix $\mathbf{P}$. This can be done simply by solving the homogeneous linear system of equations:

$$
\overline{\mathbf{x}}_{i} \cong \mathbf{P} \tilde{\mathbf{X}}_{i}
$$

where $\overline{\mathbf{x}}_{i}=\left[u_{i}, v_{i}, 1\right]$ and $\overline{\mathbf{X}}_{i}=\left[x_{i}, y_{i}, z_{i}, 1\right]$ are the homogeneous coordinates of the image pixel and $3 \mathrm{D}$ voxel in the canonical homogeneous coordinate system.

\section{Direct Computation of C-Arm Motion}

We first show that during the motion of the $\mathrm{C}$-arm, the consecutive positions of the X-ray source $\mathbf{C}=-\mathbf{R}^{t} \mathbf{T}$, in the world coordinate system can be computed without decomposing the projection matrix $\mathbf{P}$. 
Let us define the $3 \times 3$ matrix $\mathbf{p}_{13}$ and the 3 -D vector $\mathbf{p}_{4}$ such that $\mathbf{P}=$ $\left[\mathbf{p}_{13} \mathbf{p}_{4}\right]$. Using Eq. 1, we have $\mathbf{p}_{13}=\mathbf{A R}$ and $\mathbf{p}_{4}=\mathbf{A T}$. We have therefore: $\mathbf{p}_{13}^{-1} \mathbf{p}_{4}=\mathbf{R}^{-1} \mathbf{T}$. Matrix $\mathbf{R}$ is a rotation matrix and therefore $\mathbf{R}^{t}=\mathbf{R}^{-1}$.

We can then compute the position of the $\mathrm{X}$-ray source $\mathbf{C}$ without decomposing the matrix $\mathbf{P}$. We have:

$$
\mathbf{C}=-\mathbf{p}_{13}^{-1} \mathbf{p}_{4}
$$

In order to estimate the motion of the $\mathrm{C}$-arm from projection matrices $\mathbf{P}_{\boldsymbol{i}}$, the classical method $[9,7,6]$ is to decompose each projection matrix into intrinsic and extrinsic parameters. If $\mathbf{P}^{i}$ and $\mathbf{P}^{j}$ are the projection matrices obtained at two arbitrary positions of the $\mathrm{C}$-arm, the inter-frame motion is then computed:

$$
\begin{gathered}
\mathbf{P}^{i} \cong\left[\begin{array}{ll}
\mathbf{A}_{i} \mathbf{R}_{i} & \mathbf{A}_{i} \mathbf{T}_{i}
\end{array}\right] \\
\mathbf{P}^{j} \cong\left[\begin{array}{ll}
\mathbf{A}_{j} \mathbf{R}_{j} & \mathbf{A}_{j} \mathbf{T}_{j}
\end{array}\right] \\
\mathbf{R}_{(i, j)}=\mathbf{R}_{i}^{T} \mathbf{R}_{j} \\
\mathbf{T}_{(i, j)}=\mathbf{R}_{i}^{T}\left(\mathbf{T}_{j}-\mathbf{T}_{i}\right)
\end{gathered}
$$

If the imaging parameters stay constant during the C-arm motion, we show that the motion of the $\mathrm{C}$-arm can also be computed without the decomposition of the projection matrices. The motion of the C-arm $\left(\mathbf{R}_{(i, j)}, \mathbf{T}_{(\mathbf{i}, \mathbf{j})}\right)$, and the two projection matrices, $\mathbf{P}^{i}$ and $\mathbf{P}^{j}$, satisfy the following equation:

$$
\mathbf{P}^{j} \cong \mathbf{P}^{i}\left[\begin{array}{lr}
\mathbf{R}_{(i, j)} & \mathbf{T}_{(i, j)} \\
\mathbf{0}^{T} & 1
\end{array}\right]
$$

The C-arm motion is therefore directly computable, [3]:

$$
\mathbf{R}_{(i, j)}=\kappa \mathbf{p}_{13}^{i}{ }^{-1} \mathbf{p}_{13}^{j} \text { and } \mathbf{T}_{(i, j)}=\mathbf{p}_{13}^{i}{ }^{-1}\left(\kappa \mathbf{p}_{4}^{j}-\mathbf{p}_{4}^{i}\right)
$$

We may solve this system of equations by first solving for the scale factor $\kappa$. The orthogonality of the rotation matrix can be used to write:

$$
\mathbf{p}_{13}^{i} \mathbf{p}_{13}^{i{ }^{T}}=\kappa^{2} \mathbf{p}_{13}^{j} \mathbf{p}_{13}^{j}{ }^{T}
$$

This results in a least square estimation of $\kappa$. A first estimation of the rotation matrix $\tilde{\mathbf{R}}_{i j}$ can be then computed from equation 5 . This may not, however, be an orthogonal matrix. A method for finding the corresponding orthogonal matrix is proposed in the literature. This method finds the orthogonal matrix $\mathbf{R}$ as a result of the following minimization [6], [11]:

$$
\operatorname{Min}_{\mathbf{R}} \sum_{i=1 . .3}\left\|\mathbf{R v}_{i}-\mathbf{e}_{i}\right\|
$$

where $\mathbf{e}_{i}$ is the $i$ th column of the identity matrix, and $\mathbf{v}_{i}$ is the $i$ th column of the matrix $\tilde{\mathbf{R}}_{i j}^{T}$. 
This minimization is usually done using the Quaternion representation of the rotation [16], [11]. Once the scale factor $\kappa$ and then the rotation matrix $\mathbf{R}_{i j}$ are computed, the translation $\mathbf{T}_{i j}$ can be estimated from Eq. 5 .

We, however, propose a new method for computing the motion and the scale parameter from Eq.5. This method gives more accurate results compared to the two previous methods. The basic idea is to first compute the rotation matrix $\mathbf{R}_{(i, j)}$, followed by the computation of the translation $\mathbf{T}_{(i, j)}$ and the scale factor $\kappa$.

We also use the Quaternion representation of the rotation matrices in order to reduce the number of unknowns and get accurate results. The Quaternion has been used in the past to solve problems of the form $\mathbf{R u}=\mathbf{u}^{\prime}$, where $\mathbf{u}$ and $\mathbf{u}^{\prime}$ are unit vectors. Here the problem is that the unknown scalar $\kappa$ makes our equations of the form: $\mathbf{R v}=\kappa \mathbf{v}^{\prime}$. One possible solution is to normalize the vectors and write these equations in the standard form. We, however, propose a method to use the Quaternion representation to accurately compute the rotation matrix $\mathbf{R}$, by directly solving equations of the form $\mathbf{R v}=\kappa \mathbf{v}^{\prime}$. The results are more accurate than the standard minimization after normalization. This is based on a new finding on the Quaternion formulation presented in [12].

Our method of computing the rotation, followed by scale and translation is compared with computing scale first, followed by rotation and translation. Simulations and experiments with real data show that more accuracy is obtained using our method. Figure 2 shows the error in estimating the rotational angle. A number of $3 \mathrm{D}$ points from our calibration phantom are projected onto two images using two projection matrices taken from a real experiment. Gaussian noise is then added to the coordinates of each projected point. At each noise level one thousand pairs of images have been automatically generated. The projection matrices and the motion parameters are then computed. Figure 2 shows the behavior of error for the following three methods: (a) decomposition of the projection matrix, (b) direct computation of scale, followed by rotation and translation, and (c) our direct estimation of rotation first, followed by scale and translation. The decomposition of the projection matrices results in the worst estimation of the angular motion. The direct method proposed in [12] results in higher accuracy, particularly when increasing the noise level.

\subsection{Computation of C-Arm Coordinate Frame}

It is very important to establish an intuitive and natural coordinate frame within which the reconstructed volume can be presented. It was mentioned earlier that the C-arm gantry is a good frame of reference. Therefore, it is necessary to compute the X-ray source position position for each projection. This source position is assumed to move in a quasi-circular orbit. In this section we define an effective iso-center and axis of rotation for this orbit. That would be sufficient to establish the desired coordinate frame.

Once the rotations between all consecutive frames are estimated, we can compute the axis $\mathbf{r}_{i}$ and the angle $\theta_{i}$ of each rotational motion. These angles are later used in sinogram weighting[13]. Sinogram weighting means that the 


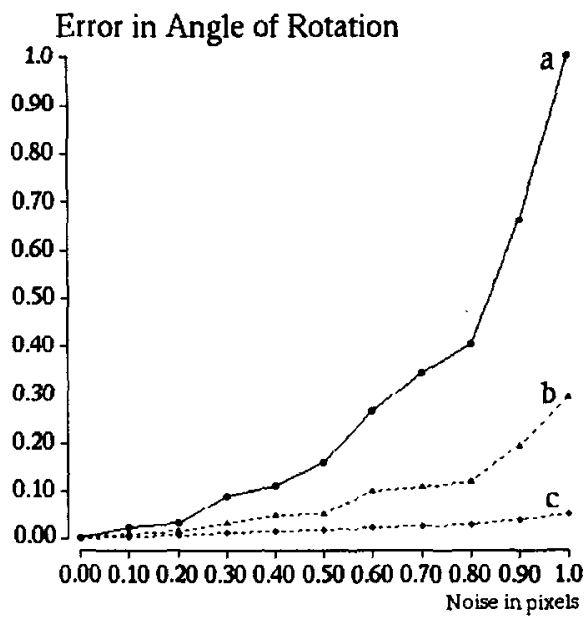

Fig. 2. The error in the estimation of angle of rotational motion from projection matrices: a) by decomposing the projection matrix, b) direct computation of scale, followed by rotation and translation, c) our direct estimation of rotation, followed by scale and translation.

projections containing corresponding data from opposing directions have to be weighted before they are filtered and backprojected into the object space.

We also need to compute the average axis of rotation. If there are $n$ frames, the average axis of rotation is:

$$
\mathbf{r}=\frac{1}{n-1} \sum_{i=1}^{n-1} \mathbf{r}_{i}
$$

where $\mathbf{r}_{i}$ is the axis of rotational motion between two consecutive frames $i$ and $i+1$.

The approximate motion of the system is then a sequence of pure rotational motion around the axis of rotation $\mathbf{r}$. In order to find the best candidate for an iso-center, we fit a cylinder, parallel to the axis of rotation, to all $\mathrm{X}$-ray source positions $\mathbf{C}_{i}, i=1 . . n$. Next, we fit a plane orthogonal to the axis of rotation $\mathbf{r}$ to all the X-ray source positions $\mathbf{C}_{i}, i=1 . . n$. The intersection of this plane with the cylinder axis is the estimated iso-center.

We now represent the projection matrices, computed in the unintuitive coordinate system of the calibration phantom, in an intuitive coordinate system with its origin at the effective iso-center and one of its coordinate axis along the average axis of rotation. Note, that this is purely a change of coordinate systems and introduces no additional errors. 


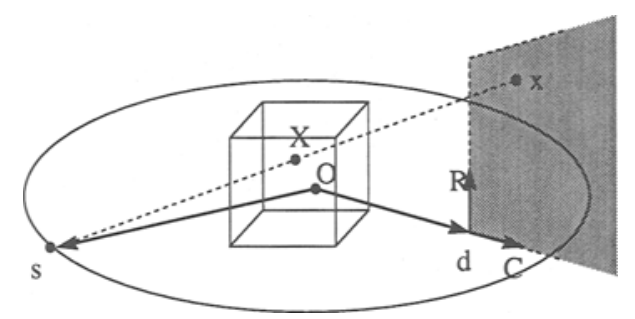

Fig. 3. Physical parameters traditionally used in the backprojection step of $3 \mathrm{D}$ cone beam reconstruction.

\section{3D Reconstruction Using Projection Matrices for Back-Projection}

$\mathrm{CT}$ reconstruction using two dimensional detectors are commonly referred to as cone-beam reconstruction techniques. In 1984 Feldkamp et. al. , [8], proposed an approximate 3D reconstruction algorithm which has been widely accepted. For an overview of exact cone beam reconstruction, refer to the work of Clack and Defrise, [1], and the work of Smith, [14]. Feldkamp reconstruction technique could be considered as a generalization of the $2 \mathrm{D}$ fan beam reconstruction extended to the third dimension. This method is based on filtered-backprojection. Stated very concisely, in this method, all the 2D projection images are filtered, backprojected into the volume and combined to form the volume data. Filteredbackprojection-type techniques including Feldkamp reconstruction have been developed in the community of CT specialists, therefore different implementations have been greatly influenced by the existing tradition. In this tradition physical parameters of the system are used in the backprojection. For the cone beam reconstruction these parameters are illustrated in Figure 3. $s$ is the X-ray source that is moving on an orbit around the center of rotation $O$. $\mathbf{d}$ is a vector connecting the center of rotation to the origin of the two dimensional detector. $\mathbf{r}$ and $\mathbf{c}$ are two unit vectors signifying the orientation of the orthogonal axes on the detector plane. A principle step of Filtered-backprojection-type cone beam reconstruction algorithms is backprojection. This consists of tracing a ray from each pixel on the image intensifier back to the X-ray source in order to mark all the $3 \mathrm{D}$ voxels which have contributed to this projection.

In this paper we are interested in a voxel oriented approach to backprojection. This approach involves tracing a ray from the $\mathrm{X}$-ray source to a voxel and continued until it intersects the image intensifier at point, marking the point by which the voxel is affected during the backprojection step. The computed tomography community is accustomed to using individual parameters of the geometry for backprojection. These parameters are computed at different calibration steps. We have designed a calibration apparatus and software that provides a transformation matrix relating each voxel in the world coordinate system to a point in the image. This matrix incorporates all the physical parameters involved in the 3-D to 2-D projection. These are the parameters that have been traditionally 
used in the backprojection step. We have successfully used the transformation matrix in the backprojection step directly, without the need to know the individual physical parameters. This approach is helpful in the following ways:

1. Eliminating the need for running separate calibration steps to compute different physical parameters.

2. Finding a mapping between the voxels to the pixels in the image plane, thereby, eliminating the effect of scale and shift caused by the image digitization process.

3. Providing a more accurate backprojection by computing all the parameters at once, keeping the overall projection error at a minimum (in a least squared sense).

4. Formulating a voxel driven backprojection method based on homogeneous transformation matrices results in an elegant and efficient algorithm.

\section{Results}

The 3D calibration and reconstruction results presented here have been obtained from prototype installations at four medical and university sites. The prototype system encompasses additional components not discussed in this paper. The geometry of the rotational angiography imaging system, a Siemens NEUROSTAR T.O.P, has been found to be reproducible. The calibration results have remained stable for over a period of more than four months. In an off-line process a calibration phantom has been positioned on the operating table, and a set radiographic images has been taken. The Pose Determination Software (PDS) has automatically made the $3 \mathrm{D}-2 \mathrm{D}$ correspondences between the makers on the calibration ring and their radiographic images. The PDS has then computed the projection matrices, the iso-center, and the inter-frame motions as described in the previous sections. The projection matrices are then transformed into the intuitive coordinate system with its origin at the estimated iso-center as described in the paper.

During the reconstruction of the patient data, only these projection matrices have been used for the back-projection process. The pixel size, image center and source to detector distances have neither been computed nor used during the calibration and reconstruction process.

Typical parameters for the prototype runs are as follows: The exposure was performed over a total angle of 200 degrees in 5 seconds, LAO to RAO; the injection rate of the contrast agent was $2.5 \mathrm{cc} / \mathrm{s}$, for a total of about $14 \mathrm{cc}$, with contrast agent being iodine ( 80 to $100 \%$ ); the XRII format was either 22 or 33 $\mathrm{cm}(9$ or 13 "), the dosage at the XRII entrance was one of two settings, 0.48 or $1.2 \mu \mathrm{Gy}(55$ or $140 \mu \mathrm{R}$ ). The dosage is about ten times lower than that for CT. However, the primary limitation of the system is not from the noise but from the low number of projections which limits the image quality. The contrast is good since the Hounsfield units are markedly above those of bone, (from 2000 to $6000 \mathrm{HU}$ ) due to the arterial injection of contrast agent. However, for the case of 


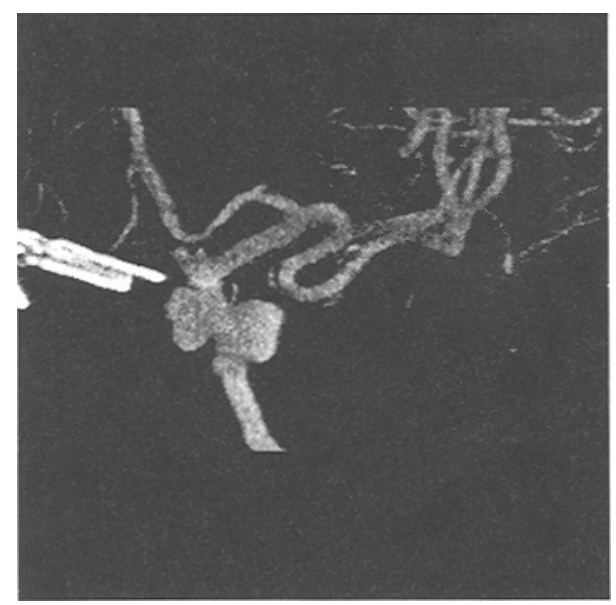

Fig. 4. Shaded-surface display image of a reconstructed volume of a human patient.

CT-Angio with venous injection, the Hounsfield units are between 200 and 300 $\mathrm{HU}$ only. The spatial resolution is approximately $0.2 \mathrm{~mm}$ "isotropic". A typical reconstruction volume is $256 \times 256 \times 256$, sometimes $512 \times 512 \times 256$ with voxel resolution being somewhere in between 0.1 to $0.3 \mathrm{~mm}$.

Figure 4 shows a portion of the shaded-surface display image of a $3 \mathrm{D}$ reconstructed volume from patient data.

\section{Conclusions}

A simple calibration and back-projection algorithm was described using projection matrices directly in a voxel driven backprojection for the reconstruction as opposed to that of computing all the geometrical parameters, including the imaging parameters. We discussed the simplicity of the entire calibrationreconstruction process, and the fact that it makes the computation of the pixel size, source to detector distance, and other explicit imaging parameters unnecessary. A robust motion estimation scheme was proposed in order to compute the motion of the $\mathrm{C}$-arm and recover the average iso-center and axis of rotation, without decomposing the projection matrices into different geometric and imaging parameters. The whole process becomes therefore quite simple. For a reproducible system, such as the NEUROSTAR T.O.P, the calibration phantom is placed and the projection matrices are computed for an exposure over a total angle of 200 degrees. This is done once every few months. For a non reproducible system the projection matrices can be computed using the patient run. The calibration ring designed to only cover a small portion of the image in this case, and does not interfere with the region of interest. This projection matrices are then directly used during the back-projection process. This calibration and reconstruction process was tested on the results obtained from prototype installation on medical sites, and the results are quite satisfactory. 


\section{References}

1. R. Clack and Michel Defrise. Overview of reconstruction algorithms for exact cone-beam tomography. pages 230-241. Proc. SPIE 2299, 1994.

2. R. Koppe E. Klotz J. Op de Beek and H. Aerts. 3D vessel reconstruction based on rotational angiography. In Computer Assitsted Radiology, pages 101-107. Springer, June 1995.

3. N. Navab et. al. Dynamic geometrical calibration for 3D cerebral angiography. pages 361-370. Proc. SPIE 2708, 1996.

4. R. Fahrig, A. J. Fox, and D. W. Holdsworth. Characterization of a C-arm mounted XRII for 3D image reconstruction during interventional neuroradiology. pages 351360. Proc. SPIE 2708, 1996.

5. R. Fahrig, M. Moreau, and D. W. Holdsworth. Three-dimensional computer tomographic reconstruction using a $\mathrm{C}$-arm mounted XRII: Correction of image intensifier distortion. Medical Physics, 24:1097-1106, 1997.

6. O.D. Faugeras. Three-Dimensional Computer Vision: A Geometric Viewpoint. MIT Press, Cambridge, MA, 1993.

7. O.D. Faugeras and G. Toscani. The calibration problem for stereo. In Proc. IEEE Conf. Comput. Vision Pattern Recog., pages 15-20, Miami, FL, June 1986. IEEE.

8. L. A. Feldkamp, L. C. Davis, and J. W. Kress. Practical cone-beam algorithm. J. Opt. Soc. Am. A, Opt. Image Sci., 1(6):612-619, June 1984.

9. S. Ganapathy. Decomposition of trnasformation matrices for robot vision. In Proc. International Conference on Robotics and Automation, pages 130-139, 1984.

10. E. Gronenschild. The accuracy and reproducibility of a global method to correct for geometric image distortion in the X-ray imaging chain. Medical Physics, 24:1875-1888, 1997.

11. R. Horaud, F. Dornaika, B. Lamiroy, and S. Christy. Object pose: The link between weak perspective, paraperspective and full perspective. Int'l J. Comput. Vision, 22(2):173-189, March 1997.

12. N. Navab. Direct estimation of rotation: A new quaternion formulation. Technical Report SCR-98-TR-631, Siemens Corporate Research, 755 College Road East, Princeton, NJ 08540, July 1998.

13. D. L. Parker. Optimal short scan convolution reconstruction for fanbeam CT. Medical Physics, 9(2):254-257, March/April 1982.

14. B. D. Smith. Cone-beam tomography: Recent advances and tutorial review. $O p$ tical Engineering, 29(5):524-534, May 1990.

15. A. Rougée A. C. Picard Y. Trousset and C. Ponchut. Geometrical calibration for 3D X-ray imaging. In Image Capture, Formatting, and Display, volume 1897, pages 161-168. SPIE, February 1993.

16. Z. Zhang and O. Faugeras. 3D Dynamic Scene Analysis: A Stereo Based Approach. Springer, Berlin, Heidelberg, 1992. 